\title{
Transitioning from population to individualized preventive cardiology
}

\author{
This article was published in the following Dove Press journal: \\ Research Reports in Clinical Cardiology \\ 9 April 2013 \\ Number of times this article has been viewed
}

\author{
Pamela B Morris' \\ Richard F Wright ${ }^{2}$ \\ 'Seinsheimer Cardiovascular Health \\ Program and Women's Heart Care, \\ Medical University of South Carolina, \\ Charleston, SC, USA; ${ }^{2}$ Heart Failure \\ Center, Pacific Heart Institute, Santa \\ Monica, CA, USA
}

\begin{abstract}
There is an ongoing discussion about whether treatment strategies developed from population based studies lead to inappropriate care of individual patients. This article proposes that despite management of lipid-lowering therapy to established low-density lipoprotein cholesterol (LDL-C) goals, significant residual risk for cardiovascular events remains in patients with established cardiovascular disease, diabetes, and metabolic syndrome (ie, central obesity, raised triglyceride levels and/or reduced high-density lipoprotein cholesterol levels). In these patients, LDL-C is often an inaccurate predictor of risk because the cholesterol content within the low-density lipoprotein particle (LDL-P) can be highly variable and thus LDL-C often inaccurately expresses an individual's likelihood of an atherosclerotic event. The LDL-P number has been found to be a better discriminator of cardiovascular risk than LDL-C in individual patients in several large epidemiologic studies that use sub-group analyses, including the Framingham Offspring Study and the Multi-Ethnic Study of Atherosclerosis. In contrast, epidemiology studies that utilize population statistics focusing on the role of lipids for initial risk assessment in entire populations do not separately evaluate these higher risk patients. The measurement of LDL-P in patients with cardiometabolic risk allows treatment of individuals through the optimization of lipid-lowering therapy to personalized goals, which would be expected to reduce that individual's risk of subsequent atherosclerotic events.
\end{abstract}

Keywords: cardiovascular disease, individualized medicine, low-density lipoprotein particles, low-density lipoprotein cholesterol

\section{Individual versus herd: which should be treated?}

In a recent analysis, Dr Eric Topol argues that the treatment of individual patients based solely on strategies developed from population based studies may lead to inappropriate care. ${ }^{1}$ He cites a recent Cochrane Collaboration review of data from 14 clinical trials in which the authors concluded that there was no net overall benefit of statin therapy in the treatment of patients without preexisting heart disease. ${ }^{2}$ For many years there has been a commonly held belief in the medical community that statin therapy should be utilized in all patients with elevated levels of low-density lipoprotein cholesterol (LDL-C) to lower that particular patient's risk of cardiovascular events. This strategy has led to potentially unnecessary treatment of people who, despite an elevated level of LDL-C, have such a low risk of such events that statin therapy would be unlikely to lead to significant individual benefit. Therefore, Dr Topol argues, such blind adherence to prespecified LDL-C population goals leads to herd-treatment, rather than targeting therapy to individual patients who are most likely to benefit. ${ }^{1}$

Estimation of risk for cardiovascular events is a dynamic area in epidemiological (population based) research. Risk estimation is an important tool in primary and
Correspondence: Pamela B Morris Seinsheimer Cardiovascular Health Program and Women's Heart Care, Medical University of South Carolina, 25 Courtenay Street ART 7063 MSC 592

Charleston, SC 29425, USA

Tel + I 8438764793

Fax + I 8438764809

Email morrispa@musc.edu
(C) 2013 Morris and Wright, publisher and licensee Dove Medical Press Ltd. This is an Open Access article which permits unrestricted noncommercial use, provided the original work is properly cited. 
secondary prevention as it can provide the impetus to initiate lifestyle changes and/or the use of an appropriate therapeutic intervention in patients while simultaneously avoiding futile treatment in those least likely to benefit. The construction of a single algorithm to predict and manage cardiovascular disease is complex when estimation from a population study is applied to an individual patient. Limitations in cardiovascular disease risk estimation could be due to: (a) the use of dated diagnostic and predictive tools, (b) the existence of unknown risk factors, (c) potential risk factors that are measured in only a subset of studies, and (d) risk factors whose association with cardiovascular disease has been difficult to reproduce. Hence, a major challenge to the health care provider is how to translate and effectively integrate population-based data into daily medical decision-making regarding an individual patient. This commentary examines the challenges with population-based approaches and makes recommendations around a personalized management approach for patients with LDL-related cardiovascular risk by highlighting how subgroup analysis can educate the treating practitioner on individual patient management decisions.

\section{Challenges with cholesterol measurements: addressing residual risk}

Epidemiologic analyses of population risk led to the establishment of LDL-C treatment goals, defined in National Cholesterol Education Program (NCEP) Adult Treatment Panel III (ATPIII) guidelines, in the hope that such treatment would lead to a reduction in cardiovascular events. ${ }^{3,4}$ Indeed meta-analyses of clinical trials have shown that LDL-C lowering therapy with statins reduces cardiovascular events in a wide range of individuals ${ }^{5,6}$ and more intensive lowering of LDL-C, at least with more aggressive use of statin therapy, further reduces the risk of occlusive vascular events. ${ }^{7}$

However, in each of these clinical trials substantial residual cardiovascular risk persisted in at-risk individuals despite such treatment. In the Get With the Guidelines analysis ${ }^{8}$ of a large cohort of patients hospitalized for cardiovascular events, only $56.5 \%$ of high risk patients with a prior history of a cardiovascular event and/or diabetes met the NCEP-ATP III goal of an on-treatment LDL-C $<100 \mathrm{mg} / \mathrm{dL}$. Moreover, only $17.5 \%$ of patients in this cohort had an on-treatment LDL-C $<70 \mathrm{mg} / \mathrm{dL}$, a goal recommended for such higher risk patients. Thus, failure to attain recommended LDL-C goals may have contributed to residual risk. Yet for patients hospitalized for a cardiovascular event without a documented history of coronary heart disease, $72.1 \%$ actually met the
NCEP-ATP III goal of an LDL-C $<130 \mathrm{mg} / \mathrm{dL}$. Even when LDL-C goals have been successfully achieved, cardiovascular disease progression and clinical events frequently occur., ${ }^{9,10}$ Hence, the Adult Treatment Panel III (ATPIII), the Canadian Cardiovascular Society/Canadian Guidelines for the Diagnosis and Treatment of Dyslipidemia, the ESC/ EAS guidelines for the management of dyslipidemias, and the National Lipid Association panel of experts, have recommended alternate LDL targets of therapy. ${ }^{11,12}$ These alternate measures to LDL-C could include non-high-density lipoprotein cholesterol (non-HDL-C), apolipoprotein B (apoB), and low density lipoprotein particle (LDL-P) number derived by nuclear magnetic resonance spectroscopy. However, in spite of these various recommendations and guidelines, the majority of the epidemiological, primary and secondary prevention trials have continued to focus on evaluating the effects of therapies on LDL-C levels from a population statistics perspective.

As a result, despite managing lipid-lowering therapy to current LDL-C goals, there is significant residual risk for cardiovascular events, particularly in patients with established cardiovascular disease, diabetes, and metabolic syndrome (ie, central obesity, raised triglyceride levels, and/or reduced high-density lipoprotein cholesterol levels). ${ }^{9}$ This underscores the difficulty in using population-based data to make daily medical decisions for individual patients.

\section{When should a new test be considered in place of the current reference standard? The role of discordance analysis}

"Gold standard" diagnostic tests often have weaknesses and yet, despite becoming obsolete, may continue to be utilized by reason of historical usage, clinical guidelines, and incorporation into previous clinical trials as key endpoints. Such an example exists for LDL-C assessment. LDL-C, apoB, and LDL-P numbers are measured primarily to ascertain LDL-attributable risk of vascular events. Experimental studies support the central role of LDL-P in atherogenesis: LDL-Ps penetrate the arterial wall and set in motion a cascade of events leading to atherosclerosis. ${ }^{13,14}$ LDL-C is a measure that quantifies the cholesterol content within a population of LDL-Ps; such cholesterol content may be highly variable over time and is dependent on disease status, diet, the use of pharmacotherapy, and other factors..$^{15}$ ApoB is the main protein component of LDL and is also found on very-low density lipoprotein and intermediate 
density lipoprotein. ApoB is measured in the clinical laboratory by several methods (immunoturbidimetric assay and the immunonephelometric assay). LDL-P quantifies the number of such particles and is independent of their cholesterol content; measurement of LDL-P number has been found to be a better discriminator of cardiovascular risk than LDL-C in several large epidemiologic studies, including the Framingham Offspring Study and the Multi-Ethnic Study of Atherosclerosis (MESA). ${ }^{16,17}$ However, this finding was only uncovered by analysis of subgroups of patients in these studies rather than by assessing the overall study populations, suggesting that LDL-P improves risk assessment and management over LDL-C in individual patients.

According to Glasziou et al, LDL-P as a potential alternative diagnostic measure must be compared to LDL-C under clinical situations in which the two tests do not agree (in such situations the two markers are thus "discordant"). ${ }^{18}$ Discordance, or non-agreement between two tests, occurs when one test detects a clinically-significant result that the other test does not. The use of a test that more accurately identifies individuals at high or low risk prevents under- or over-diagnosis of disease and thus under- or overtreatment of that disease. The finding that LDL-P is a better discriminator of cardiovascular risk than is LDL-C in subgroups of populations suggests a potential benefit to using the new test in place of, or in addition to, the reference test in individual patients.

\section{Population versus discordance studies}

In generally healthy populations with a low to moderate risk of cardiovascular disease the majority of subjects have roughly concordant levels of LDL-C and LDL-P, which dilutes potentially meaningful differences between LDL-C and LDL-P in the subgroups with discordant levels. ${ }^{17}$ Thus, studies in which only herd risk is assessed often conclude that LDL-P number is no better than traditional lipid measurements. In a study of samples obtained from participants in the Woman's Health Study, ${ }^{19}$ investigators concluded that cardiovascular disease risk prediction associated with lipoprotein (LDL-P) was comparable but not superior to that of standard lipid or apolipoprotein levels. In the EPIC-Norfolk Study, ${ }^{20}$ LDL-P and non-high-density lipoprotein cholesterol were more closely associated with coronary artery disease than LDL-C, but the association for LDL-P was lost after adjustment for high-density lipoprotein cholesterol and triglycerides. Recent findings from the Heart Protection Study, ${ }^{21}$ a study of 20,000 patients with a mean of
5.3 years of follow-up, also indicated that LDL-C, LDL-P, and apoB correlated with cardiovascular disease risk and had similar predictive value. The studies described above focused on risk of the overall population, as well as the ability to initially assess risk rather than evaluating at-risk subpopulations with discordant LDL-C and LDL-P levels.

However, studies of discordant subpopulations have yielded different results. Analyses of discordance data from both the Framingham Offspring cohort and the MESA cohort showed a high level of disagreement between LDL-C and LDL-P values on the basis of population percentiles. ${ }^{16,17}$ In the Framingham Offspring cohort, LDL-C levels were $138 \mathrm{vs} 134 \mathrm{mg} / \mathrm{dL}(P=0.09)$ in men with versus men without cardiovascular disease, but LDL-P levels were significantly different between these two groups (1641 vs $1509 \mathrm{nmol} / \mathrm{L}$ ) $(P<0.0001)$. In both studies, the cumulative incidence of cardiovascular events was significantly and substantially higher in the discordant subgroup with LDL-P > LDL-C, and significantly lower in the discordant subgroup with LDL-P $<$ LDL-C, compared with the concordant subgroup in which LDL-C and LDL-P population percentile values agreed.

The analysis of the MESA cohort reproduced in the figure below showed a higher cumulative incidence of cardiovascular events (from proportional hazards models adjusted for age and gender) in subgroups with LDL-C $<100 \mathrm{mg} / \mathrm{dL}$ and LDL-P $\geq 1060 \mathrm{nmol} / \mathrm{L}$ (adjusted rate of 11.3 events per 1000 person-years) compared with subgroups with LDL-C $\geq 100 \mathrm{mg} / \mathrm{dL}$ and LDL-P $<1060 \mathrm{nmol} / \mathrm{L}$

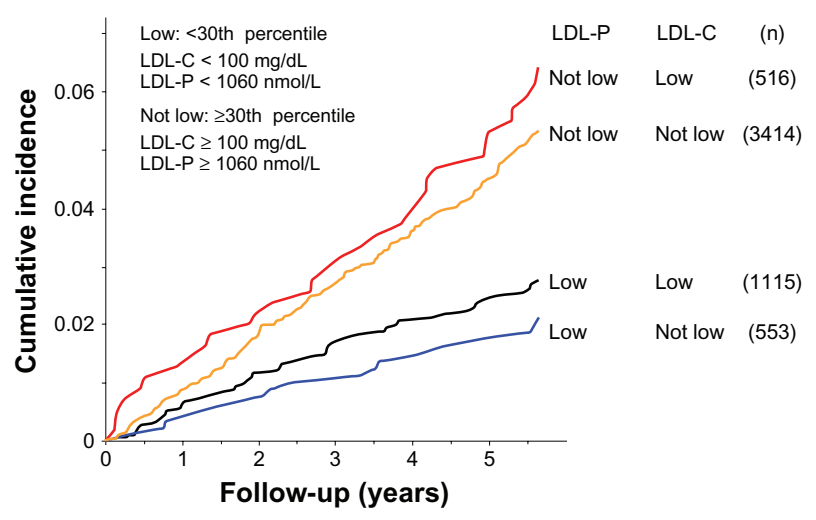

Figure I Cumulative incidence of cardiovascular events in subgroups with low LDL-C and/or low LDL-P, from proportional hazards models adjusted for age and gender.

Note: Low LDL-C and LDL-P values were defined as $<100 \mathrm{mg} / \mathrm{dL}$ and $<1060 \mathrm{nmol} / \mathrm{L}$ respectively (<30th percentile).

Reprinted from Otvos JD, Mora S, Shalaurova I, et al. Clinical implications of discordance between low-density lipoprotein cholesterol and particle number. J Clin Lipidol. 20 I ; 5(2): 105-I I3. (c) 201 I with permission from Elsevier.

Abbreviations: LDL-C, low-density lipoprotein cholesterol; LDL-P, low-density lipoprotein particle number. 
(adjusted rate of 6.2 events per 1000 person-years). ${ }^{17}$ Furthermore, a discordant subpopulation analysis from the INTERHEART study compared non-HDL-C and apoB as markers of cardiovascular risk to determine which of these markers of LDL provided more accurate risk prediction. ${ }^{22}$ The INTERHEART study is a standardized case-control study of acute myocardial infarction from 52 countries. The results from the analysis demonstrated that apoB is a more accurate marker of cardiovascular risk than non-HDL-C.

These results suggest that utilizing lipoprotein goals rather than cholesterol goals to manage cardiovascular disease risk may result in better individual patient outcomes. However, these findings cannot be reproduced using the typical population based methods - individual patient-level data must be employed. These discordant subpopulation analyses (MESA, Framingham Offspring, and the INTERHEART) have been derived from epidemiological and primary prevention studies. ${ }^{16,17,22}$ Although these studies have yielded great information, it is crucial to also run discordance subpopulation analyses in secondary prevention trials.

Finally, for an individual patient, both undertreatment and overtreatment with lipid-lowering drugs may have a significant personal, medical, and financial impact. For example, a patient at intermediate or high risk of cardiovascular disease based on Framingham Risk Score and with LDL-C $<100$ mg/dL but LDL-P > $1300 \mathrm{nmol} / \mathrm{L}$ often needs more aggressive or additional lipid-lowering therapy in order to reach a target LDL-P $<1000 \mathrm{nmol} / \mathrm{L}$, a level which was associated with lowest cardiovascular event rates in Framingham Offspring and MESA. Conversely, a patient at a similar risk level with LDL-C = $130 \mathrm{mg} / \mathrm{dL}$ but LDL-P $<1000 \mathrm{nmol} / \mathrm{L}$ may need to carefully consider whether more aggressive lipid-lowering therapy to lower LDL-C below $100 \mathrm{mg} / \mathrm{dL}$ warrants the added risks and cost of such therapy. Recent published analyses suggest that women may be at increased risk of diabetes due to statin therapy, suggesting that overaggressive statin therapy in low-risk individuals may not be advisable. ${ }^{23,24}$ Ultimately, to adequately address the question of potential cost differences between alternate measures of LDL, a cost effectiveness analysis comparing these measures in discordant subpopulations is warranted.

\section{Conclusion}

As quality of care and individual patient outcomes become more important in terms of health care and reimbursement, practitioners must have the appropriate data to optimally treat individual patients rather than the population as a whole. This is demonstrated in the case of LDL-lowering therapy, where management based on population studies has failed to adequately reduce or eliminate residual risk of cardiovascular disease events. Only through subgroup analyses do these differences become apparent, indicating that for subsets of patients with discordant LDL-C and LDL-P levels, LDL-P is the more accurate marker of cardiovascular disease risk. Likewise, in the subsets of patients with discordant non-HDL-C and apoB levels, apoB is the more accurate marker of cardiovascular disease risk. This is supported by consensus statements issued from the American College of Cardiology/American Diabetes Association and the National Lipid Association, both of which concluded that LDL-C underestimates the burden of atherogenic, cholesterol-carrying lipoproteins in patients with cardiometabolic risk, and measurements of LDL-P or apoB may more closely quantitate the atherogenic lipoprotein load. ${ }^{13,25}$ We believe the time has come for the measurement of particle number to become the standard of care for patients with cardiometabolic risk, thus allowing physicians to optimize treatment based on an individual patient's personal risk of a future cardiovascular event, rather than the risk in a population of such patients.

\section{Acknowledgments}

The authors would like to thank Erin R Baker, PhD of $\mathrm{S}^{2}$ Statistical Solutions for assistance in the development of the manuscript. $\mathrm{S}^{2}$ Statistical Solutions is a paid consultant to LipoScience, Inc.

The study was funded by LipoScience, Inc., (Raleigh, NC, USA) provider of NMR lipoprotein measurements.

\section{Disclosure}

The authors disclose the following relationships with industry: Dr Pamela B Morris is on the Speaker's Bureau of Abbott, Merck, and LipoScience, Inc. Dr Richard F Wright has received speaker honorarium from Boeringer-Ingelheim, LipoScience, and Takeda. Additionally, Dr Wright has been an expert witness for Glaxo Smith Kline and owns stock in Leading Cardiologists of America. The authors have no other conflicts of interest to declare.

\section{References}

1. Topol EJ. The Creative Destruction of Medicine: How the Digital Revolution Will Create Better Health Care. New York: Basic Books; 2012.

2. Taylor F, Ward K, Moore TH, et al. Statins for the primary prevention of cardiovascular disease. Cochrane Database Syst Rev. 2011;1: CD004816.

3. Third Report of the National Cholesterol Education Program (NCEP) Expert Panel on Detection, Evaluation, and Treatment of High Blood Cholesterol in Adults (Adult Treatment Panel III) final report. Circulation. 2002;106(25):3143-3421. 
4. Grundy SM, Cleeman JI, Merz CN, et al. Implications of recent clinical trials for the National Cholesterol Education Program Adult Treatment Panel III guidelines. Circulation. 2004;110(2):227-239.

5. Baigent C, Keech A, Kearney PM, et al. Efficacy and safety of cholesterol-lowering treatment: prospective meta-analysis of data from 90,056 participants in 14 randomised trials of statins. Lancet. 2005;366(9493):1267-1278.

6. Kearney PM, Blackwell L, Collins R, et al. Efficacy of cholesterollowering therapy in 18,686 people with diabetes in 14 randomised trials of statins: a meta-analysis. Lancet. 2008;371(9607):117-125.

7. Baigent C, Blackwell L, Emberson J, et al. Efficacy and safety of more intensive lowering of LDL cholesterol: a meta-analysis of data from 170,000 participants in 26 randomised trials. Lancet. 2010;376(9753): 1670-1681.

8. Sachdeva A, Cannon CP, Deedwania PC, et al. Lipid levels in patients hospitalized with coronary artery disease: an analysis of 136,905 hospitalizations in Get With The Guidelines. Am Heart J. 2009;157(1):111-117. e112.

9. Fruchart JC, Sacks FM, Hermans MP, et al. The Residual Risk Reduction Initiative: a call to action to reduce residual vascular risk in dyslipidaemic patient. Diab Vasc Dis Res. 2008;5(4):319-335.

10. Kones R. Primary prevention of coronary heart disease: integration of new data, evolving views, revised goals, and role of rosuvastatin in management. A comprehensive survey. Drug Des Devel Ther. 2011;5: 325-380.

11. Reiner Z, Catapano AL, De Backer G, et al. ESC/EAS Guidelines for the management of dyslipidaemias: the Task Force for the management of dyslipidaemias of the European Society of Cardiology (ESC) and the European Atherosclerosis Society (EAS). Eur Heart J. Jul 2011;32(14):1769-1818.

12. Davidson MH, Ballantyne CM, Jacobson TA, et al. Clinical utility of inflammatory markers and advanced lipoprotein testing: advice from an expert panel of lipid specialists. J Clin Lipidol. Sep-Oct 2011;5(5):338-367.

13. Brunzell JD, Davidson M, Furberg CD, et al. Lipoprotein management in patients with cardiometabolic risk: consensus statement from the American Diabetes Association and the American College of Cardiology Foundation. Diabetes Care. 2008;31(4):811-822.

14. Fredrickson DS, Levy RI, Lees RS. Fat transport in lipoproteins - an integrated approach to mechanisms and disorders. $N$ Engl J Med. 1967;276(3):148-156 contd.
15. Rosenson RS, Davidson MH, Pourfarzib R. Underappreciated opportunities for low-density lipoprotein management in patients with cardiometabolic residual risk. Atherosclerosis. 2010;213(1):1-7.

16. Cromwell WC, Otvos JD, Keyes MJ, et al. LDL particle number and risk of future cardiovascular disease in the Framingham Offspring Study - implications for LDL management. J Clin Lipidol. 2007;1(6): 583-592.

17. Otvos JD, Mora S, Shalaurova I, Greenland P, Mackey RH, Goff DC Jr. Clinical implications of discordance between low-density lipoprotein cholesterol and particle number. J Clin Lipidol. 2011;5(2):105-113.

18. Glasziou P, Irwig L, Deeks JJ. When should a new test become the current reference standard? Ann Intern Med. 2008;149(11):816-822.

19. Mora S, Otvos JD, Rifai N, Rosenson RS, Buring JE, Ridker PM. Lipoprotein particle profiles by nuclear magnetic resonance compared with standard lipids and apolipoproteins in predicting incident cardiovascular disease in women. Circulation. 2009;119(7):931-939.

20. El Harchaoui K, van der Steeg WA, Stroes ES, et al. Value of lowdensity lipoprotein particle number and size as predictors of coronary artery disease in apparently healthy men and women: the EPICNorfolk Prospective Population Study. J Am Coll Cardiol. 2007;49(5): 547-553.

21. Parish S, Offer A, Clarke R, et al. Lipids and lipoproteins and risk of different vascular events in the $\mathrm{MRC} / \mathrm{BHF}$ heart protection study. Circulation. 2012;125(20):2469-2478.

22. Sniderman AD, Islam S, Yusuf S, McQueen MJ. Discordance analysis of Apolipoprotein B and non-high density lipoprotein cholesterol as markers of cardiovascular risk in the INTERHEART study. Atherosclerosis. 2012;225(2):444-449.

23. Culver AL, Ockene IS, Balasubramanian R, et al. Statin use and risk of diabetes mellitus in postmenopausal women in the Women's Health Initiative. Arch Intern Med. 2012;172(2):144-152.

24. Sukhija R, Prayaga S, Marashdeh M, et al. Effect of statins on fasting plasma glucose in diabetic and nondiabetic patients. J Investig Med. 2009;57(3):495-499.

25. Davidson MH, Ballantyne CM, Jacobson TA, et al. Clinical utility of inflammatory markers and advanced lipoprotein testing: advice from an expert panel of lipid specialists. J Clin Lipidol. 2011;5(5):338-367.
Research Reports in Clinical Cardiology

\section{Publish your work in this journal}

Research Reports in Clinical Cardiology is an international, peerreviewed, open access journal publishing original research, reports, editorials, reviews and commentaries on all areas of cardiology in the clinic and laboratory. The manuscript management system is completely online and includes a very quick and fair peer-review system.

\section{Dovepress}

Visit http://www.dovepress.com/testimonials.php to read real quotes from published authors. 